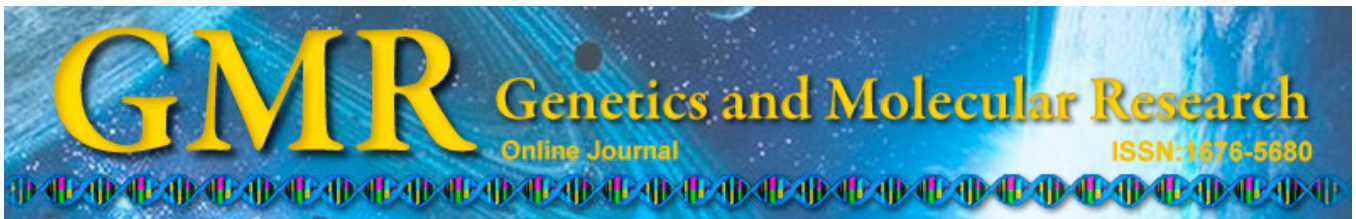

\title{
Association between GSTM1 polymorphisms and lung cancer: an updated meta-analysis
}

\author{
H. Liu, H.F. Ma and Y.K. Chen \\ Department II of Surgery, Qingdao Tumor Hospital, Qingdao, China \\ Corresponding author: Y.K. Chen \\ E-mail: yaokunchen57@yeah.net
}

Genet. Mol. Res. 14 (1): 1385-1392 (2015)

Received January 2, 2014

Accepted May 26, 2014

Published February 13, 2015

DOI http://dx.doi.org/10.4238/2015.February.13.17

\begin{abstract}
The relationship between glutathione $S$-transferase M1 (GSTM1) genetic polymorphisms and lung cancer has been reported previously. However, the results are not consistent. Therefore, to clarify the association between GSTM1 polymorphisms and lung cancer, we performed a meta-analysis based on published studies. We used the Revman 5.0 software to perform literature retrieval, article selection, data collection, and statistical analysis. We utilized a random-effect model to pool the odds ratios (ORs) and $95 \%$ confidence intervals (CIs). A total of 38 eligible studies including 5737 lung cancer patients and 6843 cancer-free control subjects were analyzed. We found no association between GSTM1 genetic polymorphisms and lung cancer risk $(\mathrm{OR}=1.15,95 \% \mathrm{CI}=0.98-1.36, \mathrm{P}=0.08)$. Including only Chinese individuals, we found no association between GSTM1 genetic polymorphisms and lung cancer risk $(\mathrm{OR}=1.13,95 \% \mathrm{CI}=0.97-1.32$, $\mathrm{P}=0.13$ ). In conclusion, we found that GSTM1 polymorphisms are not associated with lung cancer risk.
\end{abstract}

Key words: GSTM1; Lung cancer; Meta-analysis; Polymorphism 


\section{INTRODUCTION}

Recently, lung cancer has become the most common cause of cancer-related death (Persson et al., 1999). Human cancers can be initiated by DNA damage resulting from environmental factors such as alcohol use and smoking (Ada et al., 2012). However, individuals not exposed to these risk factors can also develop lung cancer, indicating that a difference in cancer susceptibility exists among individuals (Ada et al., 2012). Previous studies indicate that genetic polymorphisms in the glutathione $S$-transferase M1 (GSTM1) gene may affect the risk of lung cancer (Nakachi et al., 1993; Cao et al., 2004; Chen et al., 2004a, 2012; Atinkaya et al., 2012; Han et al., 2012; Li et al., 2012; Liang et al., 2012; Liu et al., 2012). Subjects who have inherited specific variants in the GSTM1 gene may be susceptible to the effects of chemical carcinogens and thus at a high risk of developing lung cancer. GSTM1 is a member of the glutathione $S$-transferase family and is capable of detoxifying reactive electrophiles that can act as mutagens. In addition, the GSTM1 gene is polymorphic, and several studies have reported that a deletion mutation in the GSTM1 gene results in GSTM1 deficiency associated with lung cancer (Liang et al., 2012; Liu et al., 2012; López-Cima et al., 2012; Yao et al., 2012). Seidegård et al. (1986) first reported the association between GSTM1 deficiency and lung cancer. Since then, there have been more than 40 studies examining this topic (Li et al., 2012; Liang et al., 2012; Liu et al., 2012; López-Cima et al., 2012; Yao et al., 2012). However, there is disagreement among the results of these studies. The small sample sizes used in many studies may be one explanation for this. To clarify the effect of GSTM1 polymorphisms on the risk of lung cancer, we performed a meta-analysis of 38 studies to determine the association between GSTM1 status and lung cancer risk.

\section{MATERIAL AND METHODS}

\section{Publication search}

We searched the PubMed, EMbase, Chinese Biomedical Literature Database, China National Knowledge Infrastructure (CNKI), and Wanfang databases using the search terms "lung cancer" and "GSTM1" through July 31, 2013. The online search was accompanied by a manual check of the reference lists from the identified articles and reviews for potentially eligible original reports.

\section{Inclusion criteria}

The inclusion criteria were as follows: 1) the studies examined the association between GSTM1 and lung cancer without restrictions on language or publication year; 2) the research design was a case-control study or cohort study without restrictions on age or race; 3 ) the size of the sample, odds ratios (ORs), and their 95\% confidence intervals (CIs) were provided.

\section{Exclusion criteria}

Exclusion criteria were as follows: 1) duplicate data; 2) case reports, series, abstract, comment, review, and editorial; and 3) insufficient data. 


\section{Literature quality assessment and data extraction}

We collected the following information: the name of the first author, publication year, country, number of cases and controls, and genotyping method. In a few studies, some of the data had already been reported elsewhere; therefore, only novel data were included.

\section{Data analysis}

Meta-analysis was performed using the RevMan 5.0 software, which was provided by the Cochrane Collaboration. The $Q$-test and the $I^{2}$ test were used to directly examine the heterogeneity between each study. The OR value and its $95 \% \mathrm{CI}$ was used to evaluate the relationship between GSTM1 status and lung cancer risk. To test for publication bias, we used the RevMan 5.0 statistical software to construct a funnel plot. $\mathrm{P}<0.05$ was considered to be statistically significant.

\section{RESULTS}

\section{Literature screening}

A total of 464 studies were initially identified, and 402 studies were excluded because of duplicate publication and nonclinical-based research. Eighty-three studies were reviewed, and 24 publications were excluded because they did not include control subjects. A total of 38 studies were included, all of which were case-control studies (Table 1). The 38 studies included 5737 lung cancer patients and 6843 cancer-free control subjects.

\section{Meta-analysis}

From the 38 selected studies, OR values and $95 \%$ CIs were extracted and directly used to evaluate the relationship between GSTM1 polymorphisms and lung cancer. There was heterogeneity ( $\left.<<0.001, I^{2}=77 \%\right)$ among the publications; therefore, we utilized a random-effect model to merge OR values. We found no association between GSTM1 polymorphisms and lung cancer $(\mathrm{OR}=1.15,95 \% \mathrm{CI}=0.98-1.36, \mathrm{P}=0.08$; Figure 1$)$. When only Chinese subjects were analyzed, we found no association between GSTM1 genetic polymorphisms and lung cancer risk $(\mathrm{OR}=1.13,95 \% \mathrm{CI}=0.97-1.32, \mathrm{P}=0.13$; Figure 2).

\section{Publication bias analysis}

We analyzed publication bias using the Revman 5.0 software. The funnel plots (Figures 3 and 4) show that the points were evenly distributed, symmetrical, and most were within the $95 \%$ CI. This indicates that no publication bias existed and that the study results were credible. 
Table 1. Characteristics of the studies included.

\begin{tabular}{|c|c|c|c|c|c|c|c|c|}
\hline Author & Year & Country & Genotyping methods & Cases/Controls (N) & Genotypes & Case $(\mathrm{N})$ & Genotypes & Control $(\mathrm{N})$ \\
\hline Ada & 2012 & Turkey & Multiplex PCR & $213 / 231$ & 12 & 123 & 107 & 124 \\
\hline $\mathrm{Ai}$ & 2011 & China & Multiplex PCR & $50 / 50$ & 14 & 36 & 27 & 23 \\
\hline Atinkaya & 2012 & Turkey & Multiplex PCR & $128 / 122$ & 72 & 53 & 59 & 56 \\
\hline $\mathrm{Cao}$ & 2004 & China & Multiplex PCR & $104 / 205$ & 39 & 65 & 110 & 95 \\
\hline Chang & 2006 & China & Multiplex PCR & $163 / 163$ & 57 & 106 & 85 & 78 \\
\hline Chen SD & 2004 & China & Multiplex PCR & $91 / 138$ & 56 & 35 & 73 & 65 \\
\hline Chen M & 2004 & China & Multiplex PCR & $91 / 91$ & 56 & 35 & 51 & 40 \\
\hline Chen H & 2008 & China & PCR-RFLP & $158 / 455$ & 59 & 99 & 208 & 246 \\
\hline Chen CM & 2012 & China & Multiplex PCR & $200 / 200$ & 97 & 123 & 79 & 110 \\
\hline $\mathrm{Du} \mathrm{G}$ & 2011 & China & Multiplex PCR & $125 / 125$ & 54 & 71 & 52 & 73 \\
\hline Gu YF & 2004 & China & Allele-specific PCR & $281 / 326$ & 101 & 180 & 102 & 224 \\
\hline $\mathrm{Gu}$ YF & 2007 & China & Multiplex PCR & $279 / 684$ & 164 & 115 & 325 & 359 \\
\hline Han RL & 2012 & China & Multiplex PCR & $128 / 214$ & 49 & 79 & 125 & 89 \\
\hline Lei FM & 2007 & China & Multiplex PCR & $42 / 103$ & 18 & 24 & 46 & 57 \\
\hline Li WY & 2004 & China & Allele-specific PCR & $344 / 295$ & 127 & 217 & 95 & 200 \\
\hline Li D & 2005 & China & Multiplex PCR & $156 / 93$ & 57 & 99 & 27 & 66 \\
\hline Li Y & 2006 & China & Multiplex PCR & $98 / 136$ & 44 & 56 & 61 & 39 \\
\hline Li W & 2012 & China & PCR-RFLP & $217 / 198$ & 90 & 127 & 105 & 95 \\
\hline Liang $\mathrm{KC}$ & 2012 & China & Multiplex PCR & $68 / 70$ & 21 & 47 & 31 & 39 \\
\hline Liu D & 2012 & China & PCR-RFLP & $360 / 360$ & 215 & 145 & 253 & 107 \\
\hline Liu Q & 2008 & China & Chips & $110 / 125$ & 44 & 66 & 68 & 57 \\
\hline López-Cima & 2012 & Spain & Multiplex PCR & $789 / 789$ & 375 & 401 & 358 & 418 \\
\hline $\mathrm{LuQF}$ & 2008 & China & Multiplex PCR & $56 / 42$ & 40 & 16 & 19 & 23 \\
\hline Nakachi & 1993 & Japan & Allele-specific PCR & $50 / 110$ & 19 & 31 & 57 & 53 \\
\hline Nimura & 1997 & Japan & Allele-specific PCR & $89 / 137$ & 27 & 23 & 45 & 47 \\
\hline Persson & 1999 & Sweden & Allele-specific PCR & $49 / 77$ & 17 & 32 & 20 & 57 \\
\hline Qi XS & 2008 & China & Multiplex PCR & $53 / 72$ & 34 & 19 & 41 & 31 \\
\hline Qian BY & 2006 & China & Multiplex PCR & $177 / 161$ & 69 & 108 & 53 & 108 \\
\hline Wang QM & 2006 & China & Multiplex PCR & $96 / 61$ & 40 & 56 & 19 & 42 \\
\hline Wang DQ & 2006 & China & Multiplex PCR & $91 / 91$ & 56 & 35 & 51 & 40 \\
\hline Xia Y & 2007 & China & Multiplex PCR & $58 / 131$ & 24 & 34 & 55 & 76 \\
\hline Ye WY & 2004 & China & Allele-specific PCR & $294 / 214$ & 108 & 186 & 75 & 139 \\
\hline Yao ZG & 2012 & China & PCR-RFLP & $150 / 150$ & 54 & 96 & 82 & 68 \\
\hline Ye WY & 2004 & China & Multiplex PCR & $58 / 62$ & 35 & 23 & 29 & 33 \\
\hline Zeng M & 2005 & China & Allele-specific PCR & $147 / 142$ & 56 & 91 & 51 & 91 \\
\hline Zhang JQ & 2011 & China & Multiplex PCR & $50 / 50$ & 16 & 34 & 28 & 22 \\
\hline Zhu XX & 2010 & China & Multiplex PCR & $160 / 160$ & 67 & 93 & 88 & 72 \\
\hline Zupa & 2009 & Italy & Duplex PCR & $75 / 121$ & 33 & 42 & 53 & 68 \\
\hline
\end{tabular}

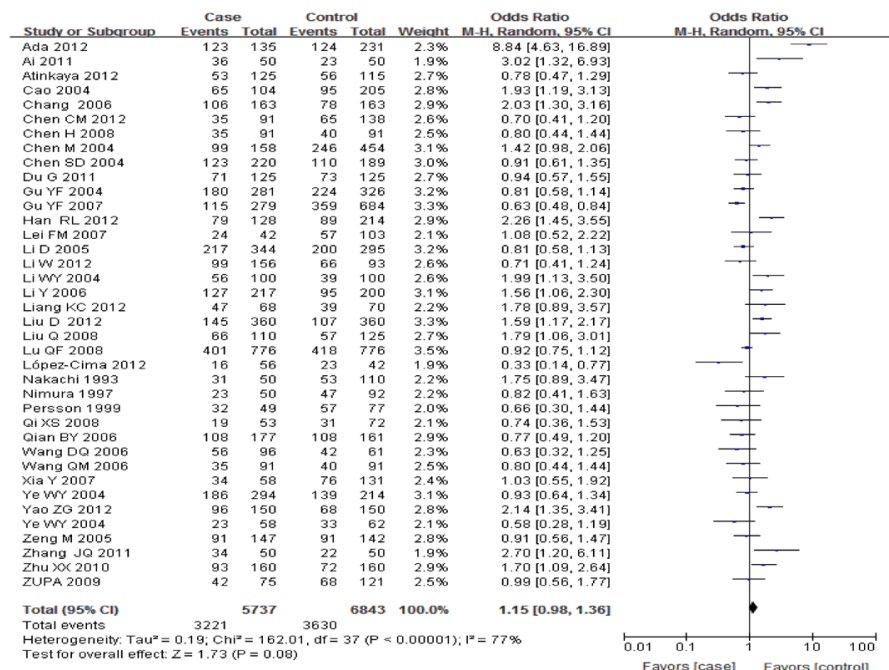

Figure 1. Forest plot of lung cancer risk associated with GSTM1 polymorphism (in total). The squares and horizontal lines correspond to the study-specific OR and $95 \% \mathrm{CI}$, respectively. The area of the squares reflects the study-specific weight. The diamond represents the pooled results of OR and 95\% CI. In this analysis, random-effect model was used. 


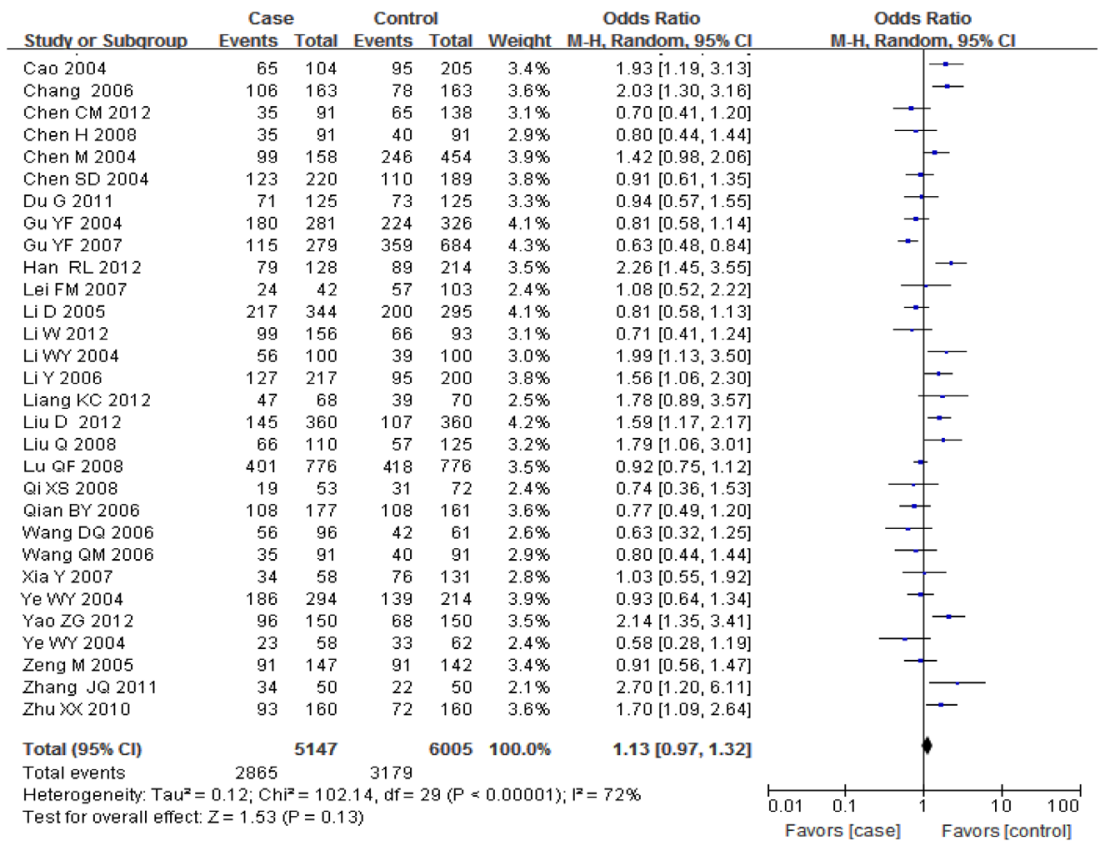

Figure 2. Forest plot of lung cancer risk associated with GSTM1 polymorphism (only in Chinese). The squares and horizontal lines correspond to the study-specific OR and 95\%CI, respectively. The area of the squares reflects the study-specific weight. The diamond represents the pooled results of OR and 95\%CI. In this analysis, random-effect model was used.

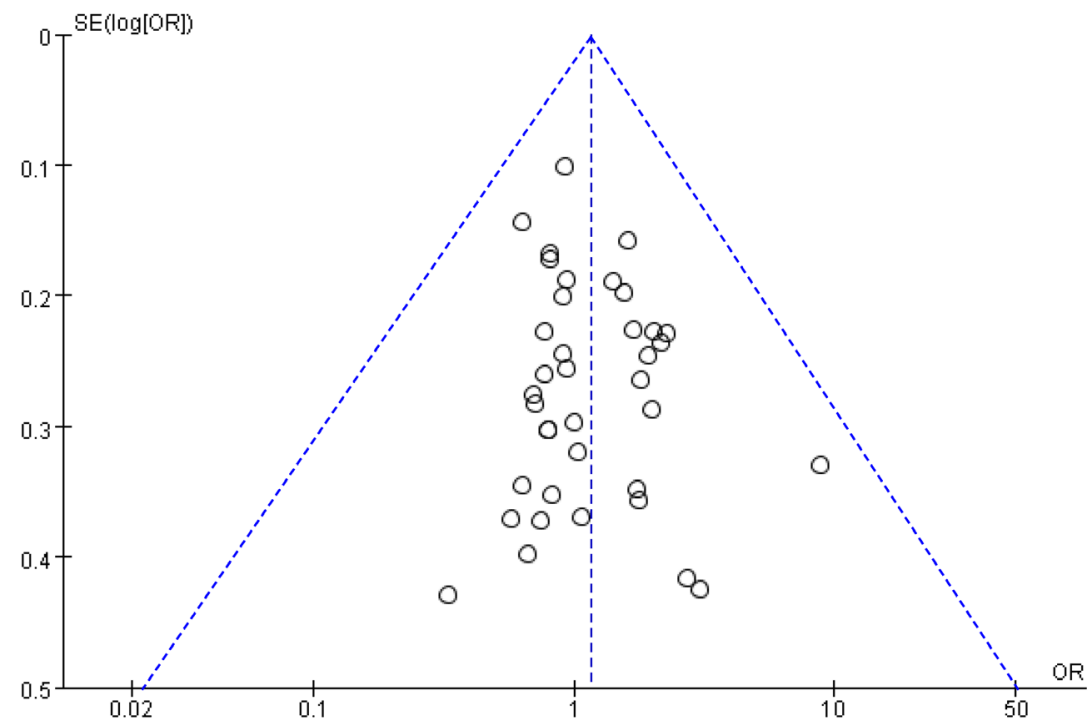

Figure 3. Funnel plot for publication bias test (in total). Each circle denotes an independent study for the indicated association. $\log [\mathrm{OR}]$, natural logarithm of OR. Horizontal line stands for mean effect size. 


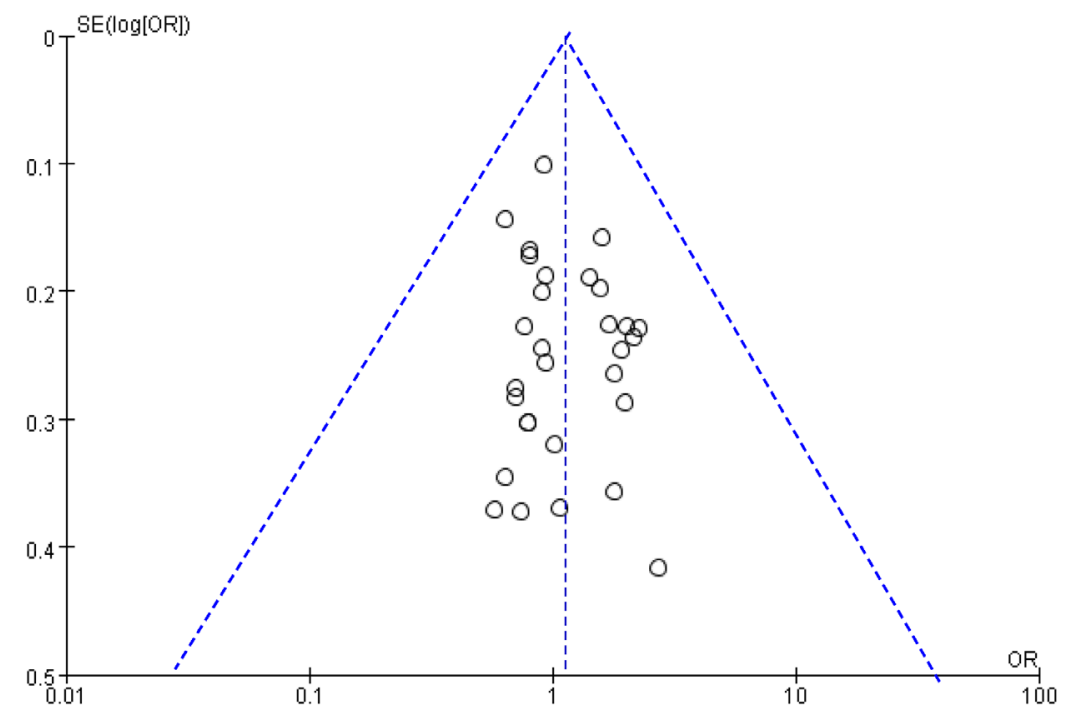

Figure 4. Funnel plot for publication bias test (only in Chinese). Each circle denotes an independent study for the indicated association. $\log [\mathrm{OR}]$, natural logarithm of OR. Horizontal line stands for mean effect size.

\section{DISCUSSION}

In the present meta-analysis, we found no association between GSTM1 polymorphisms and lung cancer risk.

The biochemical function of GSTM1 suggests a plausible biochemical rationale for individual differences in the susceptibility to certain kinds of cancer, particularly those resulting from exposure to chemical carcinogens. Individuals with deletions of both copies of GSTM1 are completely deficient in the GST isoenzyme; this enables a greater fraction of the relevant chemical carcinogens from cigarette smoke to penetrate the cellular DNA and form carcinogenic adducts. According to this model, individuals with 1 or 2 copies of the GSTM1 gene express this GST isozyme and detoxifies a greater fraction of the carcinogen or carcinogens before adduct formation. Although various previous publications have suggested that the GSTM1 polymorphism is associated with lung cancer, our meta-analysis did not show this result. In this meta-analysis, a total of 38 case-control studies were analyzed to comprehensively assess the association between GSTM1 polymorphisms and lung cancer. Genotypes in all studies were detected using genetic DNA from blood samples. Most of these studies evaluated the genotypes for quality control. The genotype distribution of controls in all studies was consistent with Hardy-Weinberg equilibrium. However, according to our meta-analysis, there is no genetic association between GSTMI and the susceptibility to lung cancer. In addition, exploring the heterogeneity between each study is an important goal of meta-analysis. In the present study, we found significant heterogeneity among the studies included, which may have resulted from the use of different genotyping methods. In addition, sensitivity analysis showed that omission of any single study did not significantly impact the combined ORs. Furthermore, the funnel plot did not reflect clear asymmetry, indicating no considerable publication bias in this meta-analysis. This indicated that our results were reliable. 
In summary, this meta-analysis of 38 studies showed that GSTM1 polymorphisms are not risk factors for lung cancer.

\section{REFERENCES}

Ada AO, Kunak SC, Hancer F, Soydas E, et al. (2012). Association between GSTM1, GSTT1, and GSTP1 polymorphisms and lung cancer risk in a Turkish population. Mol. Biol. Rep. 39: 5985-5993.

Ai C (2011). Exploring the GSTM1 gene polymorphism on the incidence of lung cancer. Contemp. Med. 17: 263.

Atinkaya C, Taspinar M, Sakiragaoglu O, Oz G, et al. (2012). The effect of CYP1A1, GSTT1 and GSTM1 polymorphisms on the risk of lung cancer: A case-control study. Hum. Exp. Toxicol. 31: 1074-1080.

Cao YF, Chen HC and Liu XF (2004). Study on the relationship between the genetic polymorphisms of GSTM1 and GSTT1 genes lung cancer susceptibility in the population of Hunan province of China. Life Sci. Res. 8: 126-128.

Chang FH, Hu TM and Wang G (2006). Relationship between CYP1A1 and GSTMl genetic polymorphisms and lung cancer susceptibility in population of Inner Mongolia. Zhonggguo Fei Ai Za Zhi 9: 413-417.

Chen CM, Jin YT, Xu HY, Zhang CY, et al. (2012). Effects of CYP1A1 and GSTM1 gene polymorphisms and BPDEDNA adducts on lung cancer. Zhonghua Yi Xue Yi Chuan Xue Za Zhi 29: 23-27.

Chen H, Yu ZC, Jin YT, et al. (2008). CYPI A1 and GSTM1 gene polymorphism on the incidence of lung cancer. Shandong Med. 48: 20-22.

Chen M, Chen SD and Wang BG (2004a). A case-control study of the impact of glutathione S-transferase M1 on the risk of lung cancer. Chin. Tumor 13: 686-688.

Chen SD, Zeng M, Li ZB, et al. (2004b). Cytochrome CYP2E1 and GST M1 and epidemiological studies: case-control study of lung cancer susceptibility. Tumor 24: 99-101.

Du G, Ma D, Tan B, Liu M, et al. (2011). Relationship between genetic polymorphism of GSTM1 gene and susceptibility to lung cancer in the population of northern Sichuan of China. Chin. Clin. Oncol. 16: 602-604.

Gu YF, Zhang SC, Lai BT, Wang H, et al. (2004). Relationship between genetic polymorphism of metabolizing enzymes and lung cancer susceptibility. Zhongguo Fei Ai Za Zhir 7: 112-117.

$\mathrm{Gu}$ YF, Zhang ZD, Zhang SC, Zheng SH, et al. (2007). Combined effects of genetic polymorphisms in cytochrome P450s and GSTM1 on lung cancer susceptibility. Zhonghua Yi Xue Za Zhi 87: 3064-3068.

Han RL, Bai TY, Chang FH, et al. (2012). GSTM1 gene polymorphism and lung cancer susceptibility in Manchu population. Central South Pharm. 10: 1-3.

Lei FM, Li SF, Zhou WD, Luo WH, et al. (2007). GSTM1 gene polymorphisms and susceptibility to lung cancer casecontrol study. Modern Prev. Med. 34: 724-726.

Li D, Zhou Q, Yuan T, Guo Z, et al. (2005). Study on the association between genetic polymorphism of CYP2E1, GSTM1 and susceptibility of lung cancer. Zhongguo Fei Ai Za Zhi 8: 14-19.

Li W, Yue W, Zhang L, Zhao X, et al. (2012). Polymorphisms in GSTM1, CYP1A1, CYP2E1, and CYP2D6 are associated with susceptibility and chemotherapy response in non-small-cell lung cancer patients. Lung 190: 91-98.

Li WY, Lai BT and Zhan XP (2004). The relationship between genetic polymorphism of metabolizing enzymes and the genetic susceptibility to lung cancer. Zhonghua Liu Xing Bing Xue Za Zhi 25: 1042-1045.

Li Y, Chen J, He X, et al. (2006). CYPIAI and GSTMI polymorphisms and susceptibility to lung cancer. J. Zhengzhou Univ. 21: 1061-1064.

Liang KC, Gan LK, Ruan L, et al. (2012). Correlational research of the relationship between the genetic polymorphisms of GSTM1 and GSTT1 in the Zhuang population and lung cancer. Acta Med. Sin. 25: 813-817.

Liu D, Wang F, Wang Q, Guo X, et al. (2012). Association of glutathione S-transferase M1 polymorphisms and lung cancer risk in a Chinese population. Clin. Chim. Acta 414: 188-190.

Liu Q, Liu J, Song B, Wang ZH, et al. (2008). Relationship between susceptibility to lung cancer and genetic polymorphism in CYPIAl and GSTM1. Shandong Med. 48: 31-33.

López-Cima MF, Alvarez-Avellón SM, Pascual T, Fernández-Somoano A, et al. (2012). Genetic polymorphisms in CYP1A1, GSTM1, GSTP1 and GSTT1 metabolic genes and risk of lung cancer in Asturias. BMC Cancer 12: 433.

Lu QF, Chen Y, Bai M, et al. (2008). Association between susceptibility of lung cancer and genetic polymorphism of GSTM1. J. Clin. Lung 13: 1444-1446.

Nakachi K, Imai K, Hayashi S and Kawajiri K (1993). Polymorphisms of the CYP1A1 and glutathione S-transferase genes associated with susceptibility to lung cancer in relation to cigarette dose in a Japanese population. Cancer Res. 53: 2994-2999.

Nimura Y, Yokoyama S, Fujimori M, Aoki T, et al. (1997). Genotyping of the CYP1A1 and GSTM1 genes in esophageal carcinoma patients with special reference to smoking. Cancer 80: 852-857. 
Persson I, Johansson I, Lou YC, Yue QY, et al. (1999). Genetic polymorphism of xenobiotic metabolizing enzymes among Chinese lung cancer patients. Int. J. Cancer 81: 325-329.

Qi XS, Lv HM, Xia Y, Shang B, et al. (2008). A primary case-control study on the relationship between genetic polymorphisms of GSTT1 and lung cancer susceptibility to the people living in high radon-exposed area. Chin. Occup. Med. 35: 361-363, 367.

Qian BY, Han HW, Gu F, et al. (2006). CYP1A1 and GSTM1 Gene polymorphism and smoking associated with susceptibility of lung cancer: a case-control study. Zhongguo Zhong Liu Lin Chuang 33: 500-503.

Seidegård J, Pero RW, Miller DG and Beattie EJ (1986). A glutathione transferase in human leukocytes as a marker for the susceptibility to lung cancer. Carcinogenesis 7: 751-753.

Wang DQ, Chen SD, Wang BG, et al. (2006a). CYP1A2, CYP2E1, and GSTM1 Gene polymorphism and susceptibility of lung cancer in Guangzhou Han population. China Oncol. 15: 578-590.

Wang QM, Lu QF, Zhen HN, et al. (2006b). CYP2C9 and GSTM1 Gene polymorphism and susceptibility of lung cancer. Zhong Liu Fang Zhi Yan Jiu 33: 8-10.

Xia Y, Sun QF, Shang B, et al. (2007). Polymorphisms of the cytochrome P450 and glutathione S-transferase genes associated with lung cancer susceptibility for the residents in high radon-exposed area. Chin. J. Radiol. Med. Prevt. 28: 327-329.

Yao ZG, Yong E, Wang HY, et al. (2012). The interacted effects between glutathione S-transferase gene polymorphism and smoking in lung cancer. C. J. Med. Guide 14: 185-187.

Ye WY, Chen Q and Chen SD (2004). Study on relationship between GSTM1 polymorphism, diet factors and lung cancer. Chin. J. Public Health 20: 1120-1121.

Zeng M, Chen SD, Xie CM, et al. (2005). Case control study on relationship between lung cancer and its susceptibility marker. Chin. J. Public Health 21: 771.

Zhang JQ, Long XY, Xiong GS, Fang LZ, et al. (2011). The relationship between glutathione S-transferase M1 and susceptibility to Xuanwei’s lung cancer. J. Kunming Med. Univ. 6: 56-58.

Zhu XX, Hu CP and Gu QH (2010). CYP1A1 polymorphisms, lack of glutathione S-transferase M1 (GSTM1), cooking oil fumes and lung cancer risk in non-smoking women. Zhonghua Jie He He Hu Xi Za Zhi 33: 817-822.

Zupa A, Sgambato A, Bianchino G, Improta G, et al. (2009). GSTM1 and NAT2 polymorphisms and colon, lung and bladder cancer risk: a case-control study. Anticancer Res. 29: 1709-1714. 